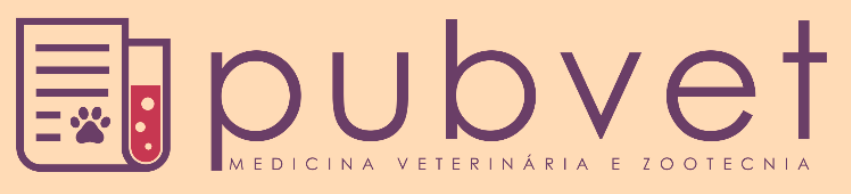

https://doi.org/10.31533/pubvet.v15n12a990.1-7

\title{
Uso da classificação ASA e concordância interobservador por médicos veterinários e estudantes no Brasil
}

\author{
Larissa Magalhães de Castro $^{1} \bullet$ (D), Sabrina Baldez Xavier $^{1} \bullet$, Rodrigo Canedo de Magalhães Dias ${ }^{1} \bullet$, \\ Bruna Pereira Gonçalves $^{1 \bullet}$, Andrei Nicolau da Costa $^{2}{ }^{\bullet}$, Eduardo Butturini de Carvalho ${ }^{2 *} \bullet$ (D) \\ ${ }^{1}$ Discente da Universidade de Vassouras, Curso de Graduação em Medicina Veterinária. Vassouras -RJ Brasil. \\ ${ }^{2}$ Docente da Universidade de Vassouras, Curso de Graduação em Medicina Veterinária. Vassouras -RJ Brasil. \\ *Autor para correspondência, E-mail: butturini@gmail.com
}

\begin{abstract}
Resumo. A classificação ASA é um sistema amplamente utilizado para classificação de estado físico do paciente cirúrgico. Todavia, estudos encontraram fraca concordância inter e intraobservador tanto em pacientes humanos como, em menor escala, em pequenos animais. Este estudo teve como objetivo investigar o perfil de uso e a variação interobservador desse sistema entre médicos veterinários e estudantes de graduação em medicina veterinária por um questionário digital com informações demográficas, sobre a formação profissional e com cinco casos clínicos elaborados. $\mathrm{O}$ formulário foi respondido por 94 participantes de 13 estados brasileiros (predominantemente RJ e SP), sendo 48,9\% profissionais graduados em 26 instituições diferentes - a maioria $(70,4 \%)$ clínicos gerais e $51,1 \%$ graduandos de 48 instituições de ensino diferentes. Entre profissionais e estudantes, $79,8 \%$ eram do sexo feminino e $20,2 \%$ do masculino, não havendo diferença na distribuição de gênero entre graduandos e graduados. Dentre os profissionais $(67,4 \%$ apenas graduados e o 32,6\% com especialização, mestrado ou doutorado), 76,1\% relataram utilizar a classificação ASA "sempre" ou "às vezes", apesar de apenas 19,6\% relatarem atuar na anestesiologia, mostrando amplo uso do sistema. A quase totalidade dos participantes relataram ter aprendido a classificação na graduação $(93,5 \%$ dos profissionais e $89,5 \%$ dos estudantes). Não foram encontradas correlações significativas entre "grau de formação" e "tempo de formado" com a utilização da classificação ASA, porém, veterinários atuantes na anestesiologia relataram uso mais frequente do sistema $(\mathrm{P}<$ 0,0001). Não houve diferença entre os escores ASA atribuídos por profissionais ou estudantes para os casos A ( $\mathrm{P}=0,263), \mathrm{B}(\mathrm{P}=0,121), \mathrm{C}(\mathrm{P}=0,443), \mathrm{D}(\mathrm{P}=0,939)$ e $\mathrm{E}(\mathrm{P}$ $=0,849)$; porém, foi encontrada fraca concordância inter observador entre os profissionais ( $\mathrm{k}$ de Fleiss $=0,365, \mathrm{P}<0,0001)$ e entre estudantes $(\mathrm{k}$ de Fleiss $=0,363, \mathrm{P}<0,0001)$. Todos os cinco casos foram classificados em no mínimo três classes diferentes por profissionais e, no caso dos estudantes, quatro dos cinco casos foram alocados em pelo menos quatro classes diferentes, mostrando grande dispersão. Este estudo mostrou fraca concordância interobservador na classificação ASA entre médicos veterinários e entre graduandos, além de demonstrar a ampla difusão e uso desse sistema de classificação de estado físico.
\end{abstract}

Palavras chave: Anestesia, cuidados pré-operatórios, veterinária

\section{ASA classification and interobserver agreement among veterinarians and graduate students in Brazil}

Abstract. ASA classification is a widespread system used for assessing physical status in surgical patients. However, studies have shown weak interobserver agreement in human and in a smaller scale in animals. This study's goal was investigating this system's usage profile and interobserver agreement among veterinarians and graduate students through an 
online survey containing demographic and professional qualification questions and five clinical cases. Ninety-four participants representing thirteen states (predominantly RJ and SP) responded the questionnaire, amongst $48.9 \%$ were veterinarians graduated in 26 different institutions - majorly general clinicians - and $51.1 \%$ were graduate students from 48 different institutions. There were $79.8 \%$ female and $20.2 \%$ male respondents and there was no difference in gender distribution between veterinarians and students. Among veterinarians (67.4\% graduates and $32.6 \%$ postgraduates), $76.1 \%$ reported using ASA classification "always" or "sometimes" although only $19.6 \%$ reported working directly in anesthesia which shows a broad usage of this system. Almost every participant reported having learned the ASA classification in graduate course $(93.5 \%$ of the veterinarians and $89.5 \%$ of students). There were no significant correlations between "study degree" and "time from graduation" and ASA status usage however veterinary anesthetists reported using it more frequently $(\mathrm{P}<0.0001)$. No difference was shown in ASA status attributed by veterinarians and students for each of the presented cases: $A(P=0,263), B(P=0.121)$, $\mathrm{C}(\mathrm{P}=0.443), \mathrm{D}(\mathrm{P}=0.939)$ and $\mathrm{E}(\mathrm{P}=0.849)$, although there was weak interobserver agreement among veterinarians (Fleiss $\mathrm{k}=0.365, \mathrm{P}<0.0001$ ) and students (Fleiss $\mathrm{k}=$ $0.363, \mathrm{P}<0.0001)$. All five clinical cases were classified in at least three different ASA classes by veterinarians and four of them were classified in at least four different ASA classes by students, showing high dispersion. This study showed weak interobserver agreement in ASA classification among veterinarians and among students besides showing a widespread usage of this physical status classification system.

Keywords: Anesthesia, preoperative care, veterinary

\section{Introdução}

O sistema de classificação ASA (do inglês American Society of Anesthesiologists) é uma escala de estado físico ordinal atribuída ao paciente na avaliação pré-operatória (Riley et al., 2014). Originalmente proposto por (Saklad, 1941) e posteriormente modificado (Dripps, 1963; Dripps et al., 1961), o sistema não permite predizer risco ou desfecho, mas sim quantificar a reserva fisiológica de um paciente (FitzHenry, 2011; Owens et al., 1978). Apesar do amplo uso e aceitação na medicina, estudos indicam baixa concordância inter e intraobservador na classificação ASA (Aronson et al., 2003; Filippi, 2000; Haynes \& Lawler, 1995; Knuf et al., 2018; Mak et al., 2002; Ranta et al., 1997; Tollinche et al., 2018). Na medicina veterinária, o sistema ASA também é amplamente utilizado, apesar de estudos escassos também indicarem subjetividade e baixa concordância interobservador (McMillan \& Brearley, 2013; Portier \& Ida, 2018). Este estudo investigou a variação interobservador da classificação ASA de cães entre médicos veterinários e estudantes de graduação de medicina veterinária.

\section{Material e métodos}

O projeto foi aprovado pelo Comitê de Ética em Pesquisa da Universidade de Vassouras (130819/2020). Um questionário digital (GoogleForms ${ }^{\circledR}$ ) foi distribuído entre 4/12/2020 e 4/3/2021 por listas de e-mails e por redes sociais para médicos veterinários e estudantes de medicina veterinária (a partir do $8^{\circ}$ período) contendo cinco casos hipotéticos de cães (Anexo I) randomizados a serem alocados nas classes I a V da classificação ASA. Além da classificação dos casos, foram coletadas informações demográficas (ano de nascimento, gênero, grau e instituição de formação, estado e cidade de atuação, ano de conclusão da graduação, área de atuação profissional, titulação de especialista) e respostas às seguintes perguntas: 1 - "Você aprendeu sobre a classificação ASA durante a graduação?" e 2 - "Você utiliza a Classificação ASA no seu dia-a-dia?". Os dados coletados foram analisados pela estatística descritiva (média, mediana, desvio padrão, percentis). Para o cálculo da correlação entre a utilização ou não da classificação ASA, entre grau de formação, "se aprendeu ou não" e tempo de formação, foi utilizada a correlação de Spearman. O teste exato de Fisher foi utilizado para calcular a diferença estatística entre a distribuição de gênero dos grupos profissionais e estudantes. O teste de Wilcoxon pareado $(\alpha=0,05)$ foi utilizado para calcular a diferença estatística entre veterinários que atuam na anestesiologia e os que não atuam, em relação ao uso da classificação ASA. O teste t não-pareado $(\alpha=$ $0,05)$ foi utilizado na comparação entre as idades dos grupos de estudantes e de profissionais. O teste de Mann-Whitney $(\alpha=0,05)$ foi usado para teste de hipóteses comparando os escores ASA atribuídos por 
profissionais ou estudantes para os casos clínicos. O kappa de Fleiss foi usado para mensurar a concordância interobservador. A análise estatística foi realizada com os softwares GraphPad Prism ${ }^{\circledR} 9.0$ e o RStudio Desktop.

\section{Resultados e discussão}

\section{Público geral respondente}

Dentre os 94 respondentes, 46 (48,9\%) eram profissionais (nenhum especialista titulado pelo Colégio Brasileiro de Anestesiologia Veterinária) e $48(51,1 \%)$ estudantes de graduação. Todos os participantes concordaram com o termo de consentimento livre e esclarecido. No total, 79,8\% dos respondentes eram do sexo feminino e 20,2\% masculino. Não houve diferença significativa na distribuição de gênero entre profissionais e estudantes $(\mathrm{P}=0,447)$; porém, houve diferença entre as idades dos grupos de estudantes e de profissionais $(\mathrm{P}<0,0001)$. Não houve diferença entre os escores ASA atribuídos por profissionais ou estudantes para os casos A ( $P=0,263), \mathrm{B}(\mathrm{P}=0,121), \mathrm{C}(\mathrm{P}=0,443), \mathrm{D}(\mathrm{P}=0,939)$ e $\mathrm{E}(\mathrm{P}=0,849)$.

\section{Médicos veterinários}

Dentre os 46 médicos veterinários graduados, 63,2\% (29/46) eram atuantes no estado do RJ, 23,7\% (12/46) em SP e 2,6\% (1/46) em cada um dos estados: MG, PR, PE, ES e RS. A média de idade dos profissionais foi de $32,3 \pm 9,2$ anos, sendo o mais jovem com 22 anos e o mais idoso com 59 anos e $76,1 \%$ (35/46) eram mulheres e 23,9\% (11/46) homens. Apenas 19,6\% (9/46) relataram atuar na anestesiologia. A maior parte dos veterinários (70,4\% - 32/46) relatou atuar em clínica geral e 11,1\% (4/46) em cirurgia e o grau de formação acadêmica mais frequente foi a graduação completa, seguida de pós-graduação lato sensu e stricto sensu (mestrado e doutorado), como mostra a figura 1.

\section{Grau de formação acadêmica}

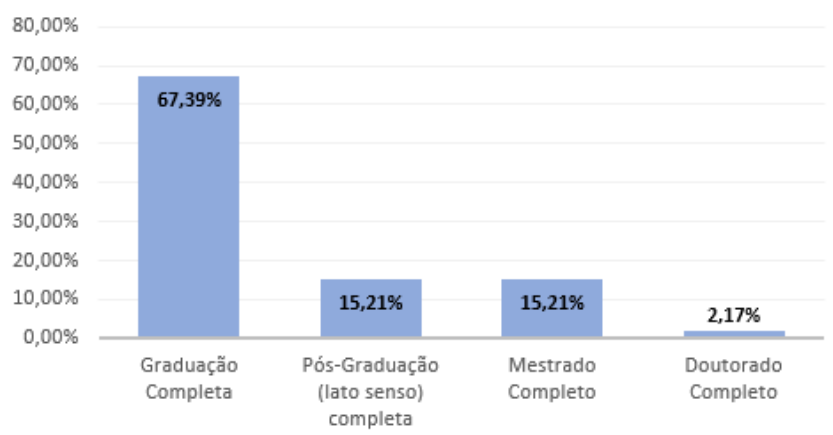

Figura 1. Demonstração de grau de formação dentre os profissionais.

Sobre o uso da classificação ASA no dia-a-dia, 34,8\% (16/46) responderam que sempre utilizam, 41,3\% (19/46) às vezes e 23,9\% (11/46) responderam que não utilizam. Apesar de veterinários atuantes na anestesiologia serem a minoria neste estudo e de muitas limitações discutidas na literatura, especialmente em relação à subjetividade do sistema (Aronson et al., 2003; Mak et al., 2002; McMillan \& Brearley, 2013; Tollinche et al., 2018), aproximadamente $3 \mathrm{em}$ cada 4 participantes relataram utilizar "sempre" ou "às vezes" a classificação, mostrando o amplo uso desse sistema na população estudada. Esse achado reforça a necessidade de ampla discussão e reforço no ensino desse sistema que ainda é fruto de muitos erros de interpretação, principalmente quando confundido como classificação de risco anestésico (Fitz-Henry, 2011; Ihejirika et al., 2015; Knuf et al., 2018; Portier \& Ida, 2018; Saklad, 1941).

Dentre os profissionais podemos identificar 26 instituições de ensino diferentes, sendo 40,4\% dos respondentes graduados em instituições públicas e 59,6\% em particulares. Quanto ao tempo de graduado, houve grande variação, desde um recém-formado até um veterinário com 34 anos de graduação (média de 6,41 \pm 9,03 anos).

Quando questionados se haviam aprendido sobre a classificação ASA durante a graduação, 93,47\% (43/46) responderam que sim e 6,52\% (3/46) afirmaram não ter aprendido sobre o sistema de classificação ASA. Este resultado indica amplo ensino deste sistema de classificação dentro da população estudada. 
Não houve correlação significativa $(\mathrm{r}=-0,109, \mathrm{P}=0,472)$ entre "grau de formação" ou "tempo de formado" ( $\mathrm{r}=-0,022, \mathrm{P}=0,886)$ com a utilização ou não da classificação ASA, indicando seu uso entre diversos perfis de formação e experiência profissional. Veterinários que afirmaram atuar na anestesiologia relataram utilizar com mais frequência classificação ASA quando comparado com veterinários que não atuam na área $(\mathrm{P}<0,0001)$. A figura 2 exibe a atribuição do escore ASA entre os profissionais.

Classificação ASA entre profissionais

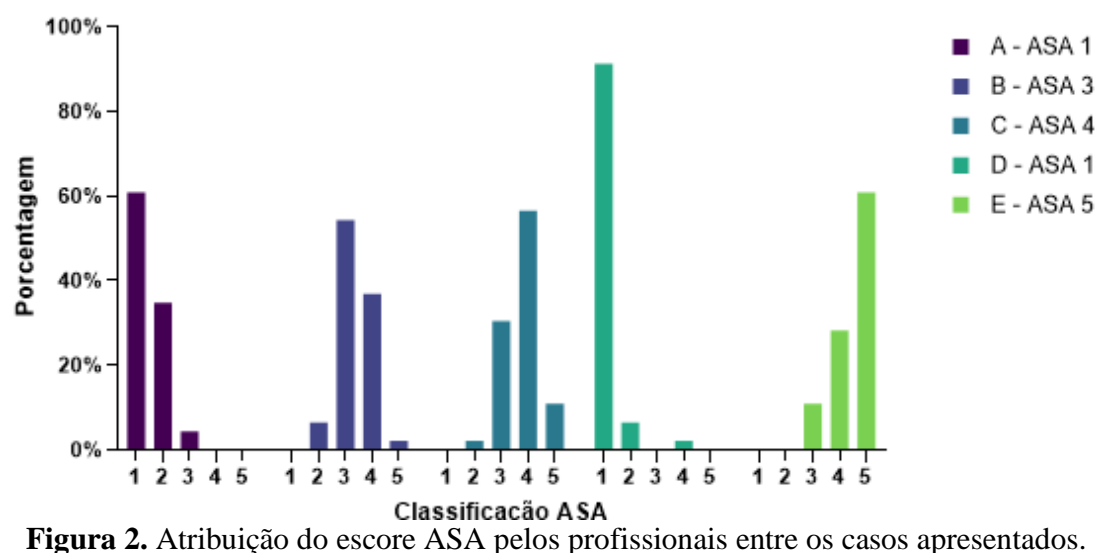

Figura 2. Atribuição do escore ASA pelos profissionais entre os casos apresentados.

Todos os casos tiveram ao menos três classificações diferentes, resultado semelhante a outro estudo com pequenos animais (McMillan \& Brearley, 2013), indicando importante dispersão na atribuição da classificação entre os profissionais, fato também observado em outros estudos na medicina (Bateman et al., 2016; Burgoyne et al., 2007; Riley et al., 2014). Houve fraca concordância interobservador entre os médicos veterinários nos cinco casos sugeridos ( $k$ de Fleiss $=0,365, \mathrm{p}<0,0001)$.

\section{Estudantes de graduação}

Foram recebidas respostas de 48 estudantes a partir do $8^{\circ}$ período da graduação, sendo 58,3\% (28/48) de instituições particulares e 41,7\% (20/48) de instituições públicas. Em relação aos estados representados, 35,4\% (17/48) dos estudantes pertencem ao estado do RJ, 18,8\% (9/48) de SP, 10,4\% (5/48) do RS, 8,30\% (4/48) de MG, 6,30\% (3/48) do CE e PR e 2,1\% (1/48) de cada um dos seguintes: SC, PI, GO, SE e PA. A média de idade dos estudantes foi de $24,6 \pm 4,6$ anos, sendo o mais novo com 19 anos e o mais idoso com 45 anos. Em relação ao gênero, 83,3\% (40/48) dos participantes estudantes eram do gênero feminino e $16,7 \%$ masculino $(8 / 40)$.

A maior parte (89,5\% - 37/48) dos estudantes afirmou ter aprendido e conhecer a classificação ASA e apenas $10,4 \%$ (11/48) responderam que não conheciam ou não aprenderam o sistema na graduação. A atribuição do escore ASA por graduandos neste estudo é detalhada na figura 3.

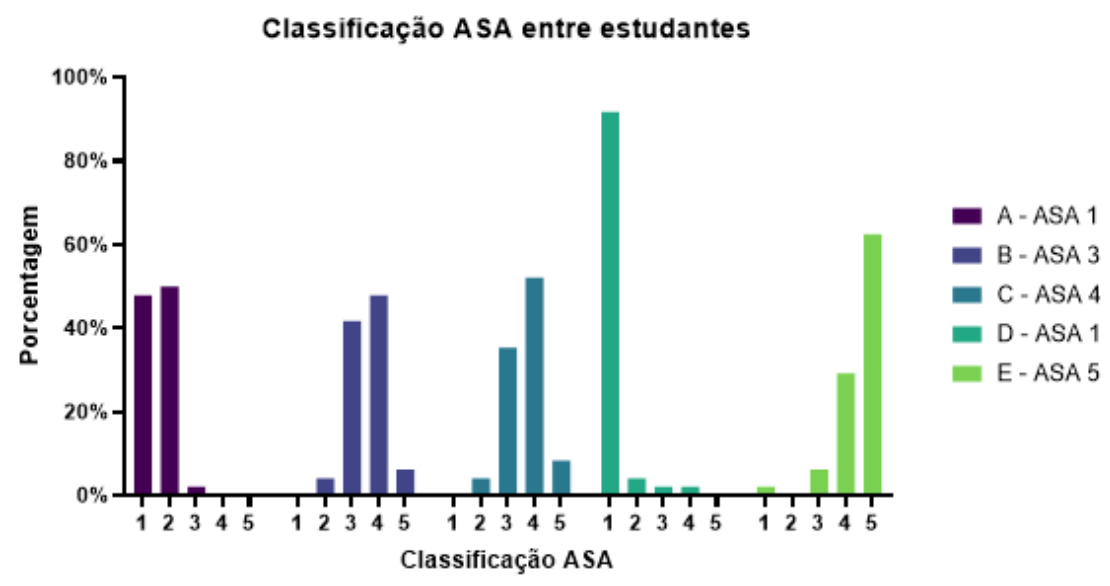

Figura 3. Atribuição do escore ASA pelos graduandos entre os casos apresentados. 
Houve importante dispersão na classificação pelos estudantes, assim como observado para a distribuição da classificação por profissionais e em diversos estudos na literatura (Aronson et al., 2003; Burgoyne et al., 2007; Ihejirika et al., 2015; Mak et al., 2002; McMillan \& Brearley, 2013; Riley et al., 2014). Todos os casos apresentaram ao menos três classificações diferentes, porém, estudantes classificaram $4 / 5$ casos em pelo menos quatro classificações diferentes, enquanto profissionais o fizeram em apenas $2 / 5$ casos. Houve fraca concordância interobservador entre os médicos veterinários nos cinco casos sugeridos $(\mathrm{k}$ de Fleiss $=0,363, \mathrm{P}<0,0001)$. Não foram encontrados estudos avaliando a variação interobservador da classificação ASA entre estudantes de medicina veterinária.

As principais limitações deste estudo são o baixo número de veterinários atuantes na anestesiologia veterinária de pequenos animais e a maior representatividade do estado do RJ nas amostras de graduandos e graduados estudadas, sendo diferenças demográficas, culturais e de treinamento, possíveis fatores de discordância na classificação ASA (Cuvillon et al., 2011).

\section{Conclusão}

Este estudo reproduziu a fraca concordância interobservador na classificação ASA entre médicos veterinários observada em trabalhos anteriores, o que indica um cuidado no uso e na interpretação desse escore. Demonstrou-se ainda, pela primeira vez e de forma similar aos profissionais, fraca concordância interobservador e considerável variação na classificação entre graduandos de medicina veterinária. Apesar da conhecida e discutida subjetividade e da baixa concordância interobservador desse sistema, o escore ASA ainda é amplamente utilizado na medicina e, como foi demonstrado na população estudada, na medicina veterinária nacional entre profissionais atuantes na anestesiologia e na clínica geral a despeito do grau e tempo de formação. Da mesma forma, o ensino da classificação ASA mostrouse bastante difundido na graduação, indicando que mesmo com amplo ensino do sistema a variação se mantém elevada.

\section{Referências bibliográficas}

Aronson, W. L., McAuliffe, M. S., \& Miller, K. (2003). Variability in the American Society of Anesthesiologists physical status classification scale. AANA Journal, 71(4), 265-276.

Bateman, R. M., Sharpe, M. D., Jagger, J. E., Ellis, C. G., Solé-Violán, J., López-Rodríguez, M., Herrera-Ramos, E., Ruíz-Hernández, J., Borderías, L., Horcajada, J., González-Quevedo, N., Rajas, O., Briones, M., Rodríguez de Castro, F., Rodríguez Gallego, C., Esen, F., Orhun, G., Ergin Ozcan, P., Senturk, E., ... Prandi, E. (2016). 36th International Symposium on Intensive Care and Emergency Medicine: Brussels, Belgium. 15-18 March 2016. Critical Care (London, England), 20(Suppl 2), 94. https://doi.org/10.1186/s13054-016-1208-6

Burgoyne, L. L., Smeltzer, M. P., Pereiras, L. A., Norris, A. L., \& Armendi, A. J. D. E. (2007). How well do pediatric anesthesiologists agree when assigning ASA physical status classifications to their patients? 1. Pediatric Anesthesia, 17(10), 956-962.

Cuvillon, P., Nouvellon, E., Marret, E., Albaladejo, P., Fortier, L.-P., Fabbro-Perray, P., Malinovsky, J.-M., \& Ripart, J. (2011). American Society of Anesthesiologists' physical status system: a multicentre Francophone study to analyse reasons for classification disagreement. European Journal of Anaesthesiology| EJA, 28(10), 742-747.

Dripps, R D. (1963). New classification of physical status. Anesthesiology, 24, 111.

Dripps, Robert D, Lamont, A., \& Eckenhoff, J. E. (1961). The role of anesthesia in surgical mortality. Jama, 178(3), 261-266.

Filippi, L. H. (2000). O eletrocardiograma na medicina veterinária. Grupo Gen-Editora Roca Ltda.

Fitz-Henry, J. (2011). Peri-operative care series. Ann R Coll Surg Engl, 93, 185-187.

Haynes, S. R., \& Lawler, P. G. P. (1995). An assessment of the consistency of ASA physical status classification allocation. Anaesthesia, 50(3), 195-199.

Ihejirika, R. C., Thakore, R. V, Sathiyakumar, V., Ehrenfeld, J. M., Obremskey, W. T., \& Sethi, M. K. (2015). An assessment of the inter-rater reliability of the ASA physical status score in the orthopaedic trauma population. Injury, 46(4), 542-546. 
Knuf, K. M., Maani, C. V, \& Cummings, A. K. (2018). Clinical agreement in the American Society of Anesthesiologists physical status classification. Perioperative Medicine, 7(1), 1-6.

Mak, P. H. K., Campbell, R. C. H., \& Irwin, M. G. (2002). The ASA physical status classification: interobserver consistency. Anaesthesia and Intensive Care, 30(5), 633-640.

McMillan, M., \& Brearley, J. (2013). Assessment of the variation in American Society of Anaesthesiologists Physical Status Classification assignment in small animal anaesthesia. Veterinary Anaesthesia and Analgesia, 40(3), 229-236.

Owens, W. D., Felts, J. A., \& Spitznagel, E. L. (1978). ASA physical status classifications: a study of consistency of ratings. The Journal of the American Society of Anesthesiologists, 49(4), 239-243.

Portier, K., \& Ida, K. K. (2018). The ASA Physical Status Classification: what is the evidence for recommending its use in veterinary anesthesia? - a systematic review. Frontiers in Veterinary Science, 5, 204.

Ranta, S., Hynynen, M., \& Tammisto, T. (1997). A survey of the ASA physical status classification: significant variation in allocation among Finnish anaesthesiologists. Acta Anaesthesiologica Scandinavica, 41(5), 629-632.

Riley, R. H., Holman, C. D. J., \& Fletcher, D. R. (2014). Inter-rater reliability of the ASA physical status classification in a sample of anaesthetists in Western Australia. Anaesthesia and Intensive Care, 42(5), 614-618.

Saklad, M. (1941). Grading of patients for surgical procedures. The Journal of the American Society of Anesthesiologists, 2(3), 281-284.

Tollinche, L. E., Yang, G., Tan, K.-S., \& Borchardt, R. (2018). Interrater variability in ASA physical status assignment: an analysis in the pediatric cancer setting. Journal of Anesthesia, 32(2), 211-218.

Histórico do artigo:

Recebido: 1 de agosto de 2021

Aprovado: 6 de setembro de 2021

Disponível on-line: 12 de novembrode 2021
Licenciamento: Este artigo é publicado na modalidade Acesso Aberto sob a licença Creative Commons Atribuição 4.0 (CC-BY 4.0), a qual permite uso irrestrito, distribuição, reprodução em qualquer meio, desde que o autor e a fonte sejam devidamente creditados 


\section{Anexo I - Casos hipotéticos apresentados aos participantes}

Caso A: Cadela castrada da raça Yorkshire, 5 anos, $4,2 \mathrm{~kg}$, submetida à cirurgia de correção de luxação da patela esquerda e retirada de nódulo cutâneo $(15 \mathrm{~mm}$ de diâmetro) para biópsia. Histórico e anamnese sem alterações dignas de nota. Exame físico: FC (Frequência Cardíaca) $=96 \mathrm{bpm}$ (Batimentos por minutos), sem alteração na ausculta cardíaca, FR (Frequência Respiratória) = $36 \mathrm{rpm}$, TPC (Tempo de Preenchimento Capilar) = 2s, turgor cutâneo 1s, mucosas normocoradas, temperatura $=38,9^{\circ} \mathrm{C}$. Exames laboratoriais sem alterações dignas de nota (Hemograma, Leucograma, ALT (Alanina aminotransferase), creatinina sérica).

Caso B: Cadela da raça Beagle, 9 anos, $5,3 \mathrm{~kg}$, castrada, submetida à anestesia para tratamento periodontal. Tutor relata seletividade para alimentação e emagrecimento recente e que o animal faz uso de benazepril, pimobendam e trilostano para tratar uma cardiopatia e a síndrome de Cushing diagnosticados há um ano. Exame físico: $\mathrm{FC}=120 \mathrm{bpm}$ com sopro holossistólico III/IV em foco mitral, FR $=64 \mathrm{rpm}$, PAS (Pressão Arterial Sistólica) $=150 \mathrm{mmHg}, \mathrm{TPC}=2 \mathrm{~s}$, turgor cutâneo $=1 \mathrm{~s}$, mucosas hiperêmicas, temperatura retal $=38,6^{\circ} \mathrm{C}$. Exames complementares: leucocitose neutrofílica sem DNNE (Desvio Nuclear de Neutrófilos à Esquerda) com eosinopenia, albumina 1,96 (2,6-3,3 g/dL); ALT 232 (21-102 U/L); fosfatase alcalina $400 \mathrm{U} / \mathrm{L}$ (20-156 U/L), ecocardiograma apresentando degeneração valvar mitral com regurgitação e turbilhonamento de fluxo transvalvar e aumento de átrio esquerdo; ECG (Eletrocardiograma) apresentando arritmia sinusal com onda p mitrale. Radiografia de tórax apresentando sinais de bronquite e aumento de câmaras cardíacas esquerdas.

Caso C: Cadela da raça Akita, inteira, 11 anos, $26 \mathrm{~kg}$, prostrada há dois dias submetida a ovariohisterectomia para tratamento de piometra aberta. Exame físico: aumento de volume abdominal com dor moderada à palpação, mucosas hipocoradas, desidratação de $8 \%$, secreção purulenta vaginal, aumento em ruídos expiratórios na ausculta pulmonar e sopro sistólico grau $\mathrm{I} / \mathrm{V}$ em foco mitral e tricúspide, $\mathrm{FC}=108 \mathrm{bpm}, \mathrm{FR}=60 \mathrm{rpm}$, temperatura retal $=40,1^{\circ} \mathrm{C}$. Exames complementares: anemia normocítica normocrômica $(\mathrm{VG}=32 \%)$, leucocitose neutrofílica com DNNE moderado, hiperlactatemia $(5,0 \mathrm{mmol} / \mathrm{L})$, hipoalbuminemia $(1,7 \mathrm{~g} / \mathrm{dL})$, hiperglicemia $(130 \mathrm{mg} / \mathrm{dL})$ e aumento da creatinina sérica $(2,0 \mathrm{mg} / \mathrm{dL})$.

Caso D: Cão inteiro sem raça definida, 2 anos, $20 \mathrm{~kg}$, submetido a orquiectomia eletiva. Histórico e anamnese sem alterações dignas de nota. Exame físico: $\mathrm{FC}=96 \mathrm{bpm}, \mathrm{FR}=46 \mathrm{rpm}, \mathrm{TPC}=2 \mathrm{~s}$, turgor cutâneo $1 \mathrm{~s}$, mucosas normocoradas, temperatura retal $38,7^{\circ} \mathrm{C}$. Hemograma, leucograma e bioquímica sérica (ALT, ureia, albumina, glicose) sem alterações dignas de nota.

Caso E: Cão castrado da raça Boxer, 8 anos, submetido à laparotomia exploratória para exérese de neoplasia esplênica com hemoperitôneo. Paciente chegou à clínica inconsciente, com pulso fraco e vazio, mucosas hipocoradas, taquicardia $(\mathrm{FC}=160 \mathrm{bpm})$, hipotensão (PAS $=60 \mathrm{mmHg})$, dispneia $(\mathrm{FR}=56 \mathrm{rpm})$. Paracentese indicou presença de sangue livre na cavidade abdominal e o paciente foi diretamente encaminhado para o centro cirúrgico. Exame coletado na admissão revelou: $\mathrm{VG}=35 \%, \mathrm{Hb}=10 \mathrm{mg} / \mathrm{dL}$, lactato $=8,3 \mathrm{mmol} / \mathrm{L}$, creatinina $=4,5 \mathrm{mg} / \mathrm{dL}$. Tutor relata histórico de cardiomiopatia dilatada tratada com digoxina há aproximadamente 2 anos. ECG na sala de emergência revelou períodos de taquicardia ventricular paroxística alternada com taquicardia sinusal com contrações ventriculares prematuras frequentes. 